

\section{DISCLAIMER}

Portions of this document may be illegible in electronic image products. Images are produced from the best available original document. 


\title{
A BRIEF HISTORY IN TIME
}

OF ION TRAPS

\section{AND THEIR ACHIEVEMENTS IN SCIENCE}

\author{
Michael H. Holzscheiter
}

Los Alamos National Laboratory

Los Alamos, NM 87545, U.S.A.

\begin{abstract}
A short history of the development of the ion storage technique for precision experiments is given. This is by no means meant to be a complete review of the field, but the intend is to use a few specific examples to describe how the persistence, ingenuity, and experimental skill of a few people has generated the core of a field which is now growing at an ever faster pace, spreading into new areas, developing sub fields, and allowing a view at nature, using very modest experimental equipment, which by far rivals even the most ambitious dreams of high energy physics.
\end{abstract}




\section{Introduction.}

When I accepted the invitation to give this presentation to an assembly of young scientists at the Nobel Symposium 91, I had not realized the complexity of the task at hand. Soon enough I found myself wandering in a forest which I could not see because of all the trees around me. But then this exact analogy opened up a view on this field which I will use to carry us on a quick, but nevertheless enjoyable, and hopefully coherent, roller coaster ride through the history of ion traps .

The angle of view I am referring to is based on the discussions in Michael McGuire's (a long standing, respected member of the "Trap Community" himself) book "An Eye for Fractals" [1]. He shows how with a minimal symmetry and very basic branching rules we can grow a wonderful tree from just a few small branches: "From a trunk grow two branches. The length of the branches are to be less than the length of the trunk, and the angles between the line of the trunk and the branches are given. Here we have a branch at $10^{\circ}, 9 / 10$ the length of the trunk and one at $60^{\circ}, 6 / 10$ the length of the trunk. Now treat the branches as trunks and do the same branching again at their ends. If at the first iteration we rotate the planes of these new branchings by $90^{\circ}$, the figure becomes three-dimensional". Figure 1 shows the result after only 10 iterations according to these rules. The resulting tree exhibits a level of symmetry and structure which is also present in the now very divers field of ion traps in the sense that even the smallest part displays a very close relation to both the origins as well as to other portions of the field, while at the same time the whole is more than just the sum of the parts.

This excursion through our field is not meant to be a rigorous and complete historical study, nor can it do justice to all the exciting experiments presently being performed in this area. I simply chose a few, very specific examples to make the point that based on the simple ideas of the original authors of long confinement times and perfect isolation for charged particles a very exciting area in physics has been developed. Due to the limited scope of this discussion I will only present the RF quadrupole traps (Paul traps) and the Penning traps, but not trapping schemes like the Kingdon trap, combined traps, and magnetic bottles. Also, while a charged particle may be represented by any entity which exhibits a non-zero charge to mass ratio, I will concentrate on the work on charged elementary particles and on ions, and will not discuss the broad field of trapping of macroscopic, particles, clusters, macromolecules, etc. 


\section{The Underlying Principle.}

The origin of this entire field can be sought in the observation that Heisenberg's Uncertainty Principle $\Delta E x \Delta T \geq h / 2 \pi$, (with $\Delta E$ being the uncertainty in the energy measurement (or definition) of an atomic transition, $\Delta T$ being the interaction time or observation time, and $\boldsymbol{h}$ being Planck's constant) simply states, that precise measurements require a long time. At the same time, for utmost precision, one desires to study an isolated system which is undisturbed by other systems surrounding it. This observation prompted Prof. Dehmelt to define the goal of obtaining a " individual particle freely floating space" [2] and embark onto the endeavor of using ion traps for precision measurement.

At the same time, an infinitely long confinement time will also allow to observe the intrinsic life time of the system, i.e. life times of metastable states or radioactive isotopes, chemical reaction times, and others. Also, the "isolation" of the particle from its environment allows a specific selection of collision partners for the study of charge exchange processes and chemical reactions, and only due to a nearly complete avoidance of reaction partners the trapping of highly charged ions as well as antimatter has become possible.

According of the picture of the field as a tree, I will develop in section 3 the roots of the ion trap development. In section 4 I will explore the "trunk" of our tree, which I define as such an area of experiments where both the long confinement times as well as the complete isolation of the particles is of significance to the results obtained. As a main example I will discuss the measurement of the electron magnetic anomaly. Section 5 discusses a few of the branches (those areas, where only one of the underlying principles is used). This area is exemplified by discussions of the external injection of charged particles into traps and by a brief description of a recent proposal to extend the use of Penning traps to extreme dense plasmas for power production via fusion. Finally a short summary and acknowledgments are given.

\section{The Early Development.}

\subsection{The Radio Frequency Quadrupole Trap.}

Based on earlier development on linear radio frequency quadrupole mass filters $[3,4]$ Prof. Wolfgang Paul, H. P. Reinhard, U. v. Zahn, [5] and E. Fisher [6] describe 
a technique with which charged particles with a specific charge-to-mass ratio e/m can be confined in all three dimensions.

To detect the ions, a resonance detection technique is used, taking advantage of the fact that for given parameters of the trap each charge-to-mass ratio exhibits a unique "eigen frequency". In addition to the radio-frequency quadrupole field an RF dipole field at the frequency $\omega_{\mathrm{RES}}$ is applied to the end caps. If through proper choice of the parameters a and $q$ (representing the amplitudes of the rf component and the dc component of the quadrupole field) the ions are brought to resonance with this dipole field the amplitude of the ion motion is increased, absorbing energy from the drive field, which can be detected. The important fact was, that different ions will have different frequencies for a given set of a and $q$, or, that at a fixed frequency one can bring all different ion species to resonance subsequently by slowly varying the $\mathrm{DC}$ potential at a constant RF amplitude. This made the quadrupole trap an ideal tool for precision mass spectrometry or residual gas analysis, areas in which RF traps have gained high respect over the last decades.

At first glance, the RF drive field seems to be a disturbance to the system, and in effect it is. Due to the continuously applied drive force stored particles are heated permanently, leading to 2 nd order doppler broadening of spectral lines. This effect can be counter acted by cooling mechanisms, either collisions with residual gas molecules, or far more powerful and selective than this, by laser cooling. Nevertheless, due to this "micro motion" the Paul trap has always been a second choice to the Penning trap if the proposal was for ultra high precision work. Recent advances have been made in this direction by using "linear" [7] or "circular" $[8,9]$ traps, in which a zero RF . amplitude can be sustained over an extended region in space, allowing laser cooling of ions to the photon recoil limit. This opens up areas which were traditionally reserved for Penning traps and the advantage of not having to supply a strong, highly homogeneous, magnetic field can be finally utilized.

\subsection{The Penning Trap.}

The fundamental idea of the Penning trap dates back to the late 1930's when F. M. Penning published his work on increasing the sensitivity of ionization vacuum gauges by using an axial magnetic field. He observes, that 'with a magnetic field of

sufficient strength, electrons, leaving the cathode, will miss the anode and return to the cathode - thereby reducing the anode current to zero, when the magnetic field is increased beyond a certain value.' He then continues to describe the effect, that '....if 
there is a sufficient number of gas molecules in the chamber, an electron can collide with these molecules. If it looses energy in these collisions the return to the cathode is impossible and the electron will describe a significantly longer path before eventually impinging onto the anode....' [10]

Even though this discussion contains the most important ingredient of the Penning trap, the long path of a charged particle in a magnetic field perpendicular to the particles momentum (and H. G. Dehmelt therefore justifiably chose the name Penning trap for this device [2]), a complete description of the confinement mechanism can be credited to J. R. Pierce [11]. In his study of the magnetron he concerned himself with the leakage of electrons from the magnetic field region. He discussed inhibiting this leakage by confining emission to a central region and adding "end hats" in axial direction. He mentions "one particular field which perhaps merits individual attention; a charge free region in which the electric potential is given by

$$
V=\frac{\omega_{0}^{2}}{2 \eta}\left(\frac{r^{2}}{2}-z^{2}\right)+V_{0}
$$

This field can be produced by hyperbolic electrodes indicated...." Figure 2 shows a copy of the relevant page in his book, and to the uninitiated reader this could be as well a page from a modern text book on confinement of charged particles. Not only does he describe the potential and the axial harmonic motion, but he also solves for the stability criterion, which essentially states that the radial confinement by the magnetic field, represented by the cyclotron frequency, must overcome the repulsion due to the radial component of the electrical quadrupole field, which is represented by the axial frequency.

\section{Precision Measurements}

With the confinement techniques mentioned above at hand, the field of precision measurements on elementary particles was off to a good start. Observation times could now be made so long, that this time scale was not limiting anymore the accuracy with which one could obtain a measurement for a frequency, and our new "artificial atom" displays a very simple level scheme (Figure 3) from which two very important and fundamental quantities can be derived. The difference between two cyclotron levels is a measure for the inertial mass of the particle(s) in the trap, and the difference between this energy splitting and the spin precession frequency gives the deviation of the electron $g$-factor from the value of 2 . But before one can set out to probe our fundamental knowledge of physics by measuring these two quantities with 
ever increasing precision, a second condition must be fulfilled. As Prof. Dehmelt points out that "....such experiments (of confining particles) are of limited value unless one also devices means for first preparing the atomic systems in a certain selected state" [2]. Keeping this important condition in mind, the real start of the field of precision measurements of the electron $\mathrm{g}$-factor started before traps even entered the picture. I will use a brief excursion of the development of this area as an example of a typical element of the "Trunk" of our tree, an area in which both the infinite confinement time as well as the complete isolation of the particles have played a crucial role in achieving the ultimate result.

\subsection{The Electron Magnetic Moment.}

According to Dirac's theory of the electron as a point like particle the $\mathrm{g}$ factor of the electron, the proportionality constant connecting spin and magnetic moment of the particle, was to be exactly 2 . This would bring the cyclotron frequency and the spin-precession frequency (Lamor frequency) to identical values and the energy levels in Figure 3 would be degenerate. Quantum electrodynamics predicts corrections to this simple picture and the $g$-factor can be redefined as $g=2(1+a)$, where a is called the anomaly, hence the expression anomalous magnetic moment of the electron. According to our simple level scheme in figure 3 one could 'simply' measure the cyclotron frequency and the Lamor frequency in separate measurements and then take the difference of these values. But because of the three orders of magnitude difference between $g$ and a one would give up accuracy (since a $10^{-5}$ measurement of $\omega_{L}$ and $\omega_{c}$ would only represent a $10^{-2}$ measurement of $\omega_{\mathrm{a}}$ ). A much higher accuracy could be achieved if the frequency $\omega_{\mathrm{a}}$ could be accessed directly with an accuracy similar to the measurement of spin and cyclotron frequency.

To first order a direct transition between the levels $|n, s=-1 / 2\rangle$ and $\ln -1, \mathrm{~s}=+1 / 2>$ is forbidden since it involves a simultaneous change of the spin quantum number and the cyclotron quantum number, therefore being a two-photon transition. But with a proper choice of the electromagnetic field configuration this transition can be driven, and the anomaly can be measured directly. A complete description of the 'experiment' now consists of: (a) trapping of the particles to allow a high accuracy despite Heisenberg's uncertainty principle, (b) preparation of an appropriate state, i.e. polarization of the sample, (c) change of spin polarization, and (d) the detection of changes of the spin polarization. As mentioned before, the zeroth order experiment (comprising only (b) - (d) was performed by H. G. Dehmelt [12]. It uses a radio-frequency discharge of a low pressure sodium gas in a magnetic field. The 
electrons in this discharge are polarized by spin-exchange with the sodium atoms, which have been polarized by optical pumping. Upon application of the proper RF drive this electron polarization changes, and these changes are detected by their effect on the sodium polarization and therefore the absorption of polarized light from the sodium lamp. The result obtained gives the ratio of the sodium magnetic moment to the magnetic moment of the electron as $g_{J} / g_{s}=1.000026 \pm 0.00003$. Since the magnetic moment for sodium atoms was known to a better accuracy, a value for the anomaly of the electron of $a_{e}=0.001116(40)$ could be extracted, making this the first experiment in a long chain to improve the accuracy of this number by many orders of magnitude. A more detailed technical report on this important measurement can also be found in reference 13 .

An extension to this method was employed by G. Gräff and his co-workers [14]. Here, for the first time, a Penning trap was utilized to store electrons at electron volt energies. These electrons were polarized by spin exchange reactions with a sodium beam from an oven which was spin polarized by passing through a hexapole magnet. The beam polarization was monitored by passing the beam through a second hexapole after it had traversed the trap. In this configuration it was not possible to monitor the electron polarization by a change of the beam polarization since the decrease in beam intensity on the detector would have been minute and not detectable in the thermal noise. Instead the spin dependence of the inelastic collision cross section for excitation of the sodium atoms by electron impact was utilized to arrive at a measurement of the electron polarization. For a fully polarized electron sample these inelastic collisions led to a certain low average energy of the electron cloud. When now the proper RF drive was applied to the electrons and their polarization was destroyed this cooling mechanism became less effective, the electron kinetic energy increased, and thereby the signal observed in an external tank circuit tuned to the axial frequency of the electrons. By slowly stepping across the $\omega_{\mathrm{a}}$ resonance and averaging the signal height over several measurements the anomaly transition could be mapped out. This experiment was repeated for a variety of trapping potentials to account for the slight dependency of the anomaly transition on the electric field, and a value for $a_{e}=0.00115966$ (30) could be reported. This value was reported as preliminary result, since many improvements to the experimental set-up had been developed, like the "flop-in" detection method for the atomic beam by using a Stern-Gerlach magnet as polarizer and a hexapole as analyzer [15], or a method to extract the electrons through an inhomogeneous magnetic field to directly detect their polarization [16], and furthermore the present value could not yet rival the results achieved in a non-trap experiment by A. Rich's group in Michigan [17] or the theoretical accuracy of $5 \times 10^{-9}$ 
at that time. But unfortunately this sequence of experiments came to an abrupt end with Prof. Gräff's untimely death.

During the same time the group around Prof. Dehmelt had been busy developing their own unique technique, which eventually lead to a "Quantum Leap" forward in this field. The crucial novelty of their approach was a consequent pursuit of the original goal to achieve a "single isolated atomic system floating at rest in free space for unlimited periods" [2]. This work resulted in the famous "Mono-Electron Oscillator" [18] where a single 1-meV electron was suspended in a Penning trap. This system has also been named by $\mathrm{H}$. G. Dehmelt as Geonium, a single electron bound to the Earth (via the trap potential), to stress the resemblance to an (ideal) atom with energy levels and transition between those levels.

The quintessence of the experiment is in two distinct advances. Firstly, being able to confine and monitor a single electron alleviates the need to prepare the sample in a specific state since the one electron always will be in a specific spin state. Secondly, by adding a very weak magnetic bottle to the highly homogeneous magnetic field a weak, spin dependent, restoring force is added to the electrical force in axial direction. This makes the axial harmonic frequency slightly dependent on the spin direction and allows to continuously monitor the spin state of the electron and therefore to observe spin flips continuously in time. When now an appropriate RF drive is applied and the frequency is stepped through the anomaly resonance the frequency of spin-flips observed will vary and a measurement of the anomaly transition is obtained. The result published in the first description of this method is $a_{e}=0.001159$ $652410(200)$, in excellent agreement with the theoretical value of the time $\left(\Delta\left(a_{\text {e }}\right.\right.$ expt $)$ $\left.a_{\text {e(theory) }}=(300 \pm 650) \times 10^{-12}\right)$. How did one get to this level of accuracy? May be the best way to display the mixture of experimental skill, ingenuity, and persistence is to follow the g-factor measurement from that time on until the presence. After having achieved a seemingly unsurpassable accuracy through the use of the well compensated Penning trap [19], which allowed continuous interaction with a single electron, while at the same time not disturbing the measurement, the next generation experiments was made possible through the observation that the magnetic bottle, limiting the precision of the data, could be omitted and be replaced by the relativistic mass shift of the electron between two adjacent spin levels [20]. This finally appeared to be a fundamental limit in accuracy. But this would be a premature conclusion. After having experimented with the scheme of a variable magnetic bottle [21] a further push towards higher accuracy was attempted by H. G. Dehmelt's group after they observed that the broadening introduced by a magnetic bottle and by the relativistic mass shift have opposite signs: 


$$
\delta \omega_{z}=\omega_{z}\left[\frac{1}{m \omega_{z}} \frac{B_{2}}{B_{o}}-\frac{1}{2 m c^{2}}\right] E_{c}
$$

and in principal the line broadening could be eliminated. Since the signal strength also follows this relation, the signal would then disappear as well, and a compromise was found in a magnetic bottle of approximately $10 \mathrm{G} / \mathrm{cm}^{2}$ for a signal width of $\delta \omega_{z}=0.13$ $\mathrm{Hz}$ [22]. Using a "trigger technique" a "micro-synchro-cyclotron" has been generated, allowing a measurement of the energy splitting of the lowest cyclotron levels $\left(\omega_{\mathrm{co}}\right)$. Inducing spin flips will shift this frequency to higher values, which can be used to determine the anomaly. Preliminary results indicate a possible precision 5 times better than previously obtained. This may be the most accurate determination of the anomalous magnetic moment to date and for the future, but then there may be no reason why a new technique should not develop, superceeding all previous methods and pushing us ahead even further

\subsection{Other "Success Stories" of the Field.}

The anomalous magnetic moment of the electron is only one amongst many success stories in the area of precision measurements using traps, and was used here by me to illustrate the force of the development made possible through the basic ingredients of infinite confinement times and perfect isolation, with a little experimental ingenuity and persistence added as spices. But there are many other measurements which followed a similar pattern:

The electron-proton mass ratio, which has first been determined in a direct measurement by the Mainz group $[23,24]$, and with the currently best value published by R. van Dyck, Jr. from the University of Washington [25] plays an important role in determining a self consistent set of fundamental constants [26]. The electron-positron magnetic moment and mass ratios [27] provide the most accurate demonstration of charged particle-antiparticle symmetry (CPT) today. A new experiment comparing the inertial masses (or to be exact the charge-to-mass ratios) of protons and antiprotons extend these CPT tests to the baryonic sector at a precision unrivaled by previous experiments [28]. The use of ion traps, both of the Penning type and the RF quadrupole type, for the realization of frequency standards with higher and higher accuracy and short and long term stability has been the back bone of the work by the group around D. Wineland at NIST [29]. Recent measurements of atomic masses aiming towards a new, atomic, mass standard to replace the antiquated standard 
kilogram have been pursued by D. Pritchard's group at MIT as well as by R. S. Van Dyck Jr. at Washington and sub-ppb accuracies have been achieved [30].

It would be far beyond the scope of this note to even describe these experiments in a general sense, especially where all the examples mentioned are being addressed at this symposium and the reader is referred to the references given as well as to the articles by the individual groups in these proceedings.

\section{The "Branches"}

Precision spectroscopy being the "trunk" of our tree, i.e. the back bone of the field of ion trapping, many new areas have been developed over the years using only part of the ultimate goals defined in the introduction. These I compare to the branches of our fractal tree, being intimately connected to the main trunk and carrying all the resemblance of a genetic heritage in them. Examples helping to define this, but which I will not discuss in any detail in this article since these will be reported on individually, are for instance the mass spectrometry of short lived isotopes [31], where the confinement techniques are used to enhance the sensitivity but the line width is determined by the internal life time of the system rather than by the storage time or the determination of the life times of metastable states [32]. Instead I want to discuss two branches in which I am personally interested and somewhat involved, the injection of external particles into traps and the studies of non-neutral plasmas, especially a technical application attempting to achieve actual power out-put through fusion.

\subsection{Injection of Particles from External Sources.}

In his early paper E. Fischer [6] made the very strong statement "...es ist unmoeglich, ein Ion von aussen in das Feld einlaufen zu lassen, - was auch ohne Rechnung verstaendlich ist, - das Ion darf erst im Raum zwischen den Elektroden aus einem neutralen Molekiul gebildet werden." (...it is impossible to introduce an ion from outside the field, - which can be understood even without calculations -, the ion must be produced in the space between the electrodes from a neutral molecule). We know very well today, that the "violation" of this law has brought us a large variety of exciting physics opportunities, amongst which we find positron physics, antiproton trapping and spectroscopy, studies of rare isotopes, retrapping of highly charged ions from the EBIT, and many more.

In the paper by $\mathrm{H}$. Schnatz et al. it is stated that "all ions studied so far in an ion trap were produced inside the harmonic well of the ion trap" [33]. In the most 
general definition of an ion, this statement was wrong. Several years before this Prof. Dehmelt's group had successfully injected positrons from an external source into a Penning trap [34]. They used radiative damping, off-axis injection, and magnetron drift enhancement of the bounce time in the trap before the 'ion' reached the entrance hole again to stabilize the particles. The efficiency of this process was only 3 in $10^{10}$, which was quite sufficient for their purpose, and is still very competitive today for direct injection of positrons into a Penning trap. In the same year as the "first" in-flight capture of ions $\mathrm{C}$. Surko had trapped positrons in a Penning type trap using buffer gas collisions to cool the positrons [35].

What is new in 'in-flight capture' is the fact that this method is more general since it does not require active cooling of the particles during the time they are traversing the trap volume, but is rather done by dynamically altering the trap potential while the particles are inside. In the true sense of the word one should only call this type of device a trap, while everything else really is a "cage". As a matter of fact, the early German literature always referred to "Käfig" rather than to "Falle", thereby recognizing the true operation of the device as a confinement system, not a catching system.

\subsubsection{Injection of Radioactive Isotopes into the "ISOLTRAP"}

The ion storage technique had so far not been used for the study of nuclear properties such as the determination of masses and moments for short lived isotopes, since these rare species had to be produced outside the trap system, cooled, eventually mass separated, and then guided with high efficiency into the trap. This was accomplished for the first time by the ISOLTRAP collaboration using an intermediate collection device in the form of an implantation foil, situated slightly inside the electrodes of a first, large volume, collection trap [33]. These foils are bombarded with a mass separated beam from the ISOLDE on-line mass separator, and, once loaded sufficiently heated to re-evaporate the ions into the collection trap. Here they are cooled using collisions with a buffer gas and then ejected as a defined bunch towards a second trap, the measurement trap. The voltage on the electrodes of this second trap is switched on at a time determined by the mean ion energy and the distance between collector and measurement trap, when the ions are inside the trap volume. To avoid spatial growth of the ion bunch during the transfer, the transfer time is minimized by accelerating the ions to approximately $1 \mathrm{keV}$ and then decelerating them again at the entrance of the second trap. An overall efficiency of this method of $10^{-4}$ was reported, with inter trap transfer efficiencies as high as $70 \%$. At the time, this method was only 
applicable to alkali ions, which can be surface ionized on the foils by heating. In the meantime different methods to inject the high energy ions into the collection trap have been developed [36] and the method can be extended to a broader variety of ion species.

A crucial consideration for the dynamics of trapping is the combination of ion velocity and bunch duration. To fit a bunch of particles inside a trap of axial extension $\mathrm{L}$ the temporal spread needs to be less than $\Delta \mathrm{T} \leq 2 \mathrm{~L} / \mathrm{v}_{\mathrm{o}}$. For the ISOLTRAP system the $30 \mu \mathrm{sec}$ pulse length of the bunch extracted from the collection trap and the $10 \mathrm{eV}$ energy spread in the bunch translate to a minimum axial dimension for the trap of 1.1 $\mathrm{cm}$, which is a quite normal dimension for standard, hyperbolic, Penning traps. But in most other cases, a standard ion trap will not suit itself to fulfilling the above condition. An important step forward in this area was therefore provided by the introduction of cylindrical open-ended Penning traps by G. Gabrielse's group [37]. This allowed to build a trap with an arbitrary large ratio of axial to radial dimension, which was, at least in the central region, highly harmonic.

\subsubsection{Trapping of Antiprotons from LEAR}

It was this possibility which allowed the capture of antiprotons from the Low Energy Antiproton Ring (LEAR) at CERN. Antiprotons are circulating in this ring (which is actually a square with a $70 \mathrm{~m}$ circumference) at energies between $1.3 \mathrm{GeV}$ and $5.9 \mathrm{MeV}$. For the trapping experiments only the lowest energy is of interest, especially since energy loss in a solid target (a foil) is used to bridge the enormous gap between the LEAR output energy and the trapping potential. LEAR can deliver both a slow, continuous spill, lasting approximately 1 hour with $10^{6}$ antiprotons $/ \mathrm{sec}$ intensity, and a fast spill, where a fraction or all of the LEAR content is extracted in a single bunch. For a typical bunch length of $200 \mathrm{~ns}$ and a desired maximum energy of $30 \mathrm{keV}$ a length of the trap of $50 \mathrm{~cm}$ is required, allowing for some jitter and rise time in the voltage switching, if one wants to capture all the antiprotons in the energy bin of $0-30$ $\mathrm{keV}$. Smaller traps at lower potentials can be used, if the maximum number is not of critical concern to the experiment, and the successful work in trapping and cooling several tens of thousand antiprotons by the PS196 collaboration [38] is exemplifying this. For experiment PS200 [39] as well as for many possible applications of ultra-low energy antiprotons for nuclear and atomic physics measurements [40] it is paramount to capture as many antiprotons as possible in a trap, and following the above course of arguments a trap of a total length of $50 \mathrm{~cm}$ was constructed. The trap structure consists of 7 electrodes: the entrance foil, a central region comprised of five cylinders ( 
2 endcaps, 2 compensation electrodes, and the central ring) with lengths and diameters carefully chosen to produce a harmonic, orthogonalized, quadrupole potential in the central region [37], and a cylindrical high voltage exit electrode. For the purpose of the initial antiproton capture the trapping region is defined by the entrance foil and the high voltage exit electrode. This region of the trap has no harmonic properties and will be referred to as the "catching trap" throughout this paper. The central, harmonic region serves a dual purpose: to initially hold cold electrons in preparation for the electron cooling, and then to collect the cooled antiprotons after the electron cooling has taken place. This trap is instrumented with two independent tuned circuits for the detection of electrons and antiprotons via the signals induced in the compensation rings and for resistively cooling the antiprotons after the electrons have been ejected. An incoming beam pulse from LEAR will loose energy in the entrance electrode, which acts as a degrading foil, and if the energy of the beam is carefully tuned by changing the upstream density of material to be traversed, a maximum number of antiprotons below $30 \mathrm{keV}$ will emerge from the down stream side - theoretical predictions are calling for $2.8 \%$ [41] - and travel towards the exit electrode. Here they are reflected by the applied potential, but before they can reach the degrading foil again, the potential on this entrance electrode is quickly ramped up and the particles are confined. In recent test experiments up to approximately 1 million antiprotons have been captured in this way and have been electron cooled to low energies within a few minutes. Figure 4 shows a schematic of the trap and a sample of an energy spectrum of captured antiprotons before electron cooling. More details on this experiment are given elsewhere in these proceedings [42].

\subsection{Plasma Studies in Ion Traps}

Another area in which traps have entered an area of physics where they were not expected to play an important role at first sight is the field of plasma physics. Leaving the idea of a single particle freely floating in space behind and pushing for the maximum number of particles which possibly can be put in a trap collective behaviour starts to play a more and more important role. One can ask the question on how many particles one can pack into the trap volume and by the simple consideration that the radially confining magnetic force must overcome the repulsive electrical force due to both the applied external potential and the internal space charge potential one arrives at a maximum density (the Brillouin density) of approximately $2.7 \times 10^{9} \mathrm{~B}^{2} 1 / \mathrm{cm}^{3}$, where $B$ is the magnetic field in Tesla. Densities at this limit have been achieved, mostly by loading a cloud and then spinning up its rotation with an external torque. Conservation 
of angular momentum will then lead to a compression of the cloud to the maximum density [43].

This system allows a detailed study of the dynamics of non-neutral plasmas and helps to understand instabilities in different confinement scenarios, to minimize loss mechanism, and to investigate specific fusion processes. But for all practical purposes aiming at power production, the total reactivity per unit volume is severly limited and the question was posed, how to extend this technique beyond the brillouin density limit.

\subsubsection{Ion Traps vs. Fusion Machines}

Considering the enormous amounts of money being put into the development of tokamak reactors for fusion energy despite the fact that the cost per incremental progress is growing exponentially, one may argue that alternate fusion approaches should be pursued if (a) they do not require magnetic confinement superior to tokamaks, (b) their physics basis may be experimentally tested, (c) they offer near-term applications to important technical problems, and (d) their cost to proof-of-principle is low enough to be consistent with nowadays budget constraints. Lead by these principles D. C. Barnes and collaborators [44] have asked the natural question whether the excellent confinement observed in non-neutral (single species) plasmas stored in Penning traps might be applied to the controlled release of nuclear fusion energy. Equally naturally one may arrive at the conclusion that the reactivity per unit volume one can obtain from such systems is very much too low to be of practical importance. This low reactivity is associated with the Brillouin limit which constrains the plasma mass energy density ( $\mathrm{nMc}^{2}$, where $\mathrm{n}$ is the number density, $\mathrm{M}$ is the mass, and $\mathrm{c}$ is the velocity of light) relative to the magnetic field energy density $\left(B_{0}{ }^{2} / 2 \mu_{0}, B_{0}\right.$ is the magnetic induction and $\mu_{0}$ is the permeability of free space). On the other hand, it is an important observation that the Brillouin density limit only applies in a volume average sense, and local densities far beyond the Brillouin density could be achievable if a steep spatial density gradient could be imposed to the cloud. To realize this density gradient it is proposed to inject low energy, low $\mathrm{P}_{\Theta}$ (canonical angular momentum) electrons into a spherical Penning trap. These electrons are accelerated radially inward to approximately $100 \mathrm{keV}$ by the effective spherical well (comprising the applied potential, the space charge potential, and the magnetic field). Convergence of these particles near $r=0$ produces a dense, inertially confined core. This core represents a virtual cathode, and if neutral gas atoms are admitted to the system, they will be inonized, attain thermonuclear energies, and are electromagnetically confined in the 
virtual cathode formed by the electron core. To maintain the stable, non-thermal state which is important to achieve the density gradient required, a constant throughput of particles will be provided. Electrons will be injected near $r=0$ through a small hole in the "polar region" of the spherical well. Electrons will fall down into the well and get deflected at the central core and will (in the absence of collisions) undergo a number of transits in the well until they return to the injection hole and can escape. This confinement time is typically given by the transit time and the ratio of the hole area to the surface of the spheroid, and is for typical parameters of this proposal in the order of $10^{-8} \mathrm{sec}$, which is several orders of magnitude shorter than the beam-beam collision times. A schematic of this experiment is shown in figure 5.

To test the fundamental principle of this idea, we have designed and constructed a small Penning trap $(r=3 \mathrm{~mm})$ which will be operated at several 10's of $\mathrm{kV}$ in a ultra-high vacuum system. Electrons from $\mathrm{a} \mathrm{LaB}_{6}$ field emitter will be focussed through a $100 \mu \mathrm{m}$ entrance hole and generate a beam-like distribution in the spherical well. Initial diagnostic of the density of the core will be obtained by relaxing the potential at the opposite end-cap after a predetermined time, which will let the beam traverse through the trap.

If a significant density at the center of the trap has been obtained, the beam will be deflected radially and the beam spot on a detector in the fringe magnetic field (for initial amplification) will be enlarged compared to the empty trap case. Besides this initial diagnostics for the fundamental principle of the proposed method, a number of technical issues like high voltage stand-offs and corona discharges from surfaces, avoidance of filling the trap with a thermal background charge from ionizing residual gas molecules, alignment requirements, etc. will be addressed at the same time. One specifically interesting challenge lies in the fact, that in contrast to standard trap experiments, where only a tiny fraction of the trap volume near the center is being utilized and where highly harmonic electrostatic potentials and extremely homogeneous magnetic fields can be readily maintained, here a spherical volume which nearly touches the electrode surfaces will be utilized. To achieve a harmonic potential in this volume with relative deviations from the ideal case being less than $10^{-3}$ while at the same time allowing for truncated electrodes and injection and ejection holes, careful electrostatic modelling has been used to generate a specific surface shape of the electrodes. Monitoring axial and radial modes of (low density) plasmas generated in this trap the harmonicity of the actual potential can be probed. We expect to be able to answer these and many other technical questions in our test system in the next few years, and will then be able to shed light on the question: "Can we build a fusion 
reactor for everybody's garage". Should this be achievable, Penning traps will have bridged the giant gap between infinitely small densities ( a single particle floating freely in empty [ $10^{-17}$ Torr] space) and the highest plasma densities obtainable in terrestial machines ( $10^{6}$ times the Brillouin limit).

\section{Summary and Conclusions}

These few selected examples have shown how diverse the field of ion trapping has become in the few decades since the first trapping experiment. I have by far not covered all areas, and therefore have only shown a small portion of the tree. Additionally, an area which has been not at all the topic of this talk, or even the symposium, which certainly has been influenced by the ion trap work, is the development of traps for neutral particles. May be one could compare the atom trap field to a seedling which has sprung from the original tree, even though it has in the meantime by far out grown (at least in volume) the ion trap community. Common to both fields, and to all the individual experiments, is the relatively small size, both physically and fiscally, of these experiments, some of which are testing territory which has been the traditional playing ground for larger and larger high energy accelerators. The next generation accelerators are finding more and more fiscal constraints and the energy increase one can imagine at this time is very limited, and it is a valid argument to look for "garage type" (weren't they used to be called "table-top not too long ago?) experiments, which can shed light on questions far beyond the reach of accelerators. Excellent examples for these types are the g-2 measurements, which may hint to a substructure of the electron [45] or the measurement of the gravitational acceleration of the antiproton [46], probing physics at the Planck mass scale. 


\section{References:}

1. Michael D. McGuire; "An Eye for Fractals"; Addison Wesley (1991)

2. H. G. Dehmelt, Adv. At. Mol. Phys. 3 \& 5, 53 (1967)

3. W. Paul, H. Steinwedel; Z. Naturforschung 8a, 448 (1953)

4. W. Paul, M. Raether, Z. Physik 140, 262 (1955)

5. W. Paul, H. P. Reinhard, U. v. Zahn; Z. Physik 152, 143 (1958)

6. E. Fischer; Z. Physik 156, 1 (1959)

7. M. G. Raizen, J. M. Gilligan, J. C. Bergquist, W. M. Itano, D. J. Wineland; Journ. Mod. Opt. 39, 233 (1992)

8. D. A. Church; J. Appl. Phys. 40, 3127 (1969) and

9. I. Waki, S. Kassner, G. Birkl, H. Walther; Phys. Rev. Lett. 68, 2007 (1992)

10. F. M. Penning; Physica Scripta 3, 873 (1936)

11. J. R. Pierce; "Theory and Design of Electron Beams", D. van Nostrand co., NY, chapter 3 (1949)

12. H. G. Dehmelt, Phys. Rev. 109, 381 (1958)

13. H. G. Dehmelt, F. L. Walls; Phys. Rev. Lett. 21, 127 (1968)

14. G. Gräff, E. Klempt, G. Werth; Z. Physik 222, 201 (1969)

15. M. Holzscheiter, G. Gräff; Phys. Rev. Lett.79A, 380 (1980)

16. G. Gräff, H. Kalinowsky, J. Traut; Z. Physik A297 (1980) 35

17. A. Rich, J. Wesley; Rev. Mod. Phys. 44, 250 (1972)

18. D. Wineland, P. Ekstrom, H. G. Dehmelt; Phys. Rev. Lett. 31, 1279 (1973)

19. R. S. Van Dyck Jr, D. Wineland, P. Ekstrom, H. G. Dehmelt; Appl. Phys. Lett. 28, 446 (1976)

20. L. S. Brown, G. Gabrielse; Rev. Mod. Phys. 58, 233 (1986)

21. R. S. Van Dyck, Jr., F. L. Moore, D. L. Farnham, P. B. Schwinberg; Rev. Sci. Instrum. 57, 593 (1986)

22. R. K. Mittelman, F. L. Palmer, H. G. Dehmelt; Hyperfine Int. 81, 105 (1993)

23. G. Gärtner, E. Klempt; Z. Physik A287, 1 (1978)

24. G. Gräff, H. Kalinowsky, J. Traut, Z. Physik A297, 35 (1980)

25. R. S. Van Dyck et. al, Int. J. Mass Spec. Ion Proc. 66, 327 (1985)

26. R. S. Van Dyck, Jr., F. L. Moore, D. L. Farnham, P. B. Schwinberg; Int. J. of Mass Spec. \& Ion Proc. 66, 327 (1985)

27. R. S. Van Dyck, Jr.. P. B. Schwinberg, H. G. Dehmelt; Phys. Rev. Lett. 59, 26 (1987)

28. G. Gabrielse, X. Fei, L. A. Orozco, R. L.Tjoelker; Phys. Rev, Lett. 65, 1317 (1990); see also D. Phillips; these proceedings

29. W. Itano, J. C. Bergquist, D. J. Wineland; Science 237, 612 (1987)

30. see both the reports by D. Prichard and R. S. van Dyck, Jr. in these proceedings.

31. G. Bollen, these proceedings

32. D. Church, these proceedings

33. H. Schnatz, G. Bollen, P. Dabkiewicz, P. Egelhoff, F. Kern, H. Kalinowsky, L. Schweikhard, H. Stolzenberg; NIM A251, 17 (1986)

34. H. G. Dehmelt, P. B. Schwinberg, R. S. Van Dyck, Jr.; Phys. Rev. Lett. 47, 1679 (1981)

35. C. M. Surko, et al.; in "Positron Studies of Solids, Surfaces, and Atoms"; p221 (1986)

36. R. B. Moore; Hyperfine Int. 81, 45 (1993) 
37. G. Gabrielse, L. Haarsma, S. L. Rolston; Int. J. Mass. Spec. Ion Proc. 88, 319 (1989)

38. G. Gabrielse, X. Fei, L. A. Orozco, R. L. Tjoelker, J. Haas, H. Kalinowsky, T. A. Trainor, W. Kells; Phys. Rev. Lett. 63, 1360 (1989)

39. M. H. Holzscheiter, et al.; Nucl. Phys A558, 709c (1993)

40. M. H. Holzscheiter, P. L. Dyer, S. Hoibraten, N. S. P. King, R. A. Lewis, D. C. Lizon, G. L. Morgan, J. Rochet, M. M. Schauer, J. A. Schecker, T. Otto; "The PS200 Catching Trap - A New Tool for Ultra-Low Energy Antiproton Physics"; to be publisched in the 'Proceedings of the Third Biennial Conference on Nucleon-Antinucleon Physics, NAN '93 in Moscow, Russia, Sept.

41. M. H. Holzscheiter, Physica Scripta, 46, 272 (1992)

42. M. Holzscheiter, these proceedings

43. J. J. Bollinger, D. J. Heinzen, F. L. Moore, Wayne M. Itano, D. J. Wineland, Daniel H. E. Dubin; Phys. Rev. A48, 525 (1993)

44. D.C. Barnes, R. A. Nebel, Leaf Turner; submitted to Physics of Fluids (1993)

45. H. G. Dehmelt; Hyperfine Int. 81, 1 (1993)

46. T. Goldman, et al.; Hyperfine Int. 81, 87 (1993) 


\section{Figure Captions:}

Figure 1: A tree can be grown by very simple replacement rules (taken with the author's permission from Michael D. Mc. Guire "An Eye for Fractals"; Addison Wesley (1991)).

Figure 2: Copy of the relevant page in J.R. Pierce's book on the "Theory and Design of Electron Beams", describing the Penning trap confinement scheme.

Figure 3: Energy level scheme of a single particle confined in a Penning trap.

Figure 4: (a) The PS 200 antiproton catching trap set-up, and (b) a sample spectrum of antiprotons captured from a single LEAR pulse.

Figure 5: Schematic of the proposed Penning trap system for fusion studies. 
After ten iterations, view from the front

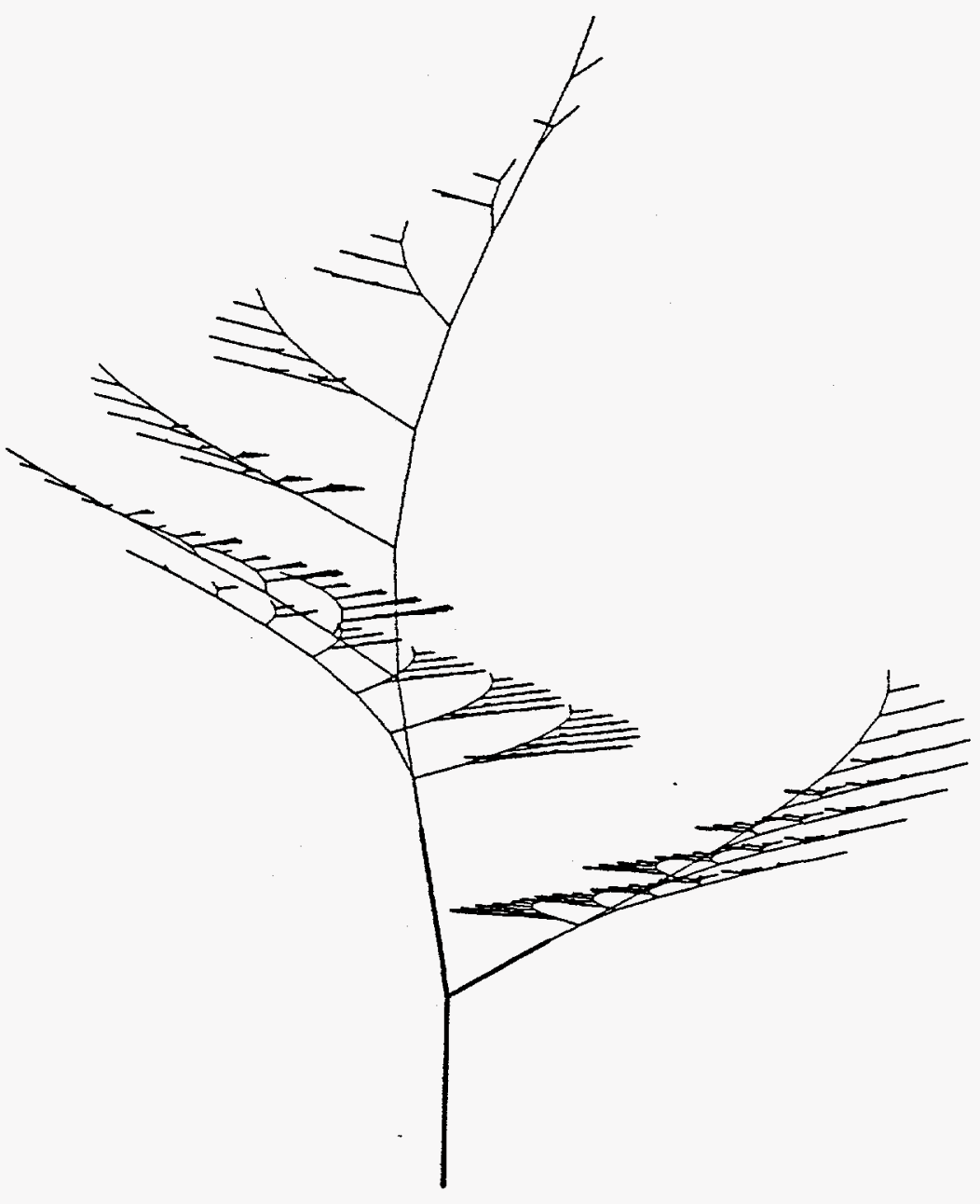


We see from the above example that a strong magnetic field can "trap" electrons so that they can move only so far in either the $r$ or the $z$ directions. There is one particular field which perhaps merits individual attention; a charge-free region in which the electric potential is given by

$$
V=\frac{\omega_{0}^{2}}{2 \eta} \cdot\left(\frac{r^{2}}{2}-z^{2}\right)+V_{0}
$$

This field can be produced by hyperbolic electrodes indicated in cross-section in Fig. 4.7. Suppose we have also a uniform magnetic field of strength $B$ in the $z$ direction. There can be no magnetic forces in the $z$ direction, so the equation of motion in this direction is

$$
z=-\omega_{0}^{2} z
$$

This implies a sinusoidal oscillation of radian frequency $\omega_{0}$. If it were not for the magnetic field, electrons would escape in the $r$ direction. We see that, ... remote from the axis, the maximum potential (that at $z=0$ ) is closely given by

$$
V=\frac{\omega_{0}^{2} r^{2}}{4 \eta}
$$

From (4.28), remote from the axis the cutoff potential approaches

$$
V=\frac{\omega_{0}^{2} r^{2}}{8 \eta}
$$

Thus, the condition under which

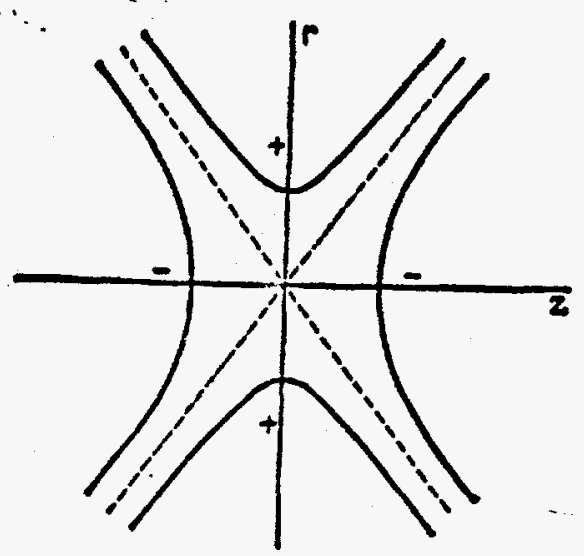

Fio. 4.7-Electron motion between hy. perbolic electrodes may be limited to a certain region.by use of an axial magnetic field. electrons cannot get indefinicely far away from the axis is that

$$
\omega_{0}>\sqrt{2} \omega_{0}
$$

We see that it is possible to obtain a pure sinusoidal motion of electrons trapped in this combination of electric and magnetic fields. 
n $-1 / 2$

$+1 / 2$

n

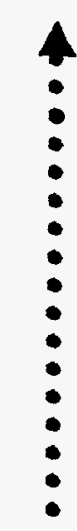

$\hat{1}$

2

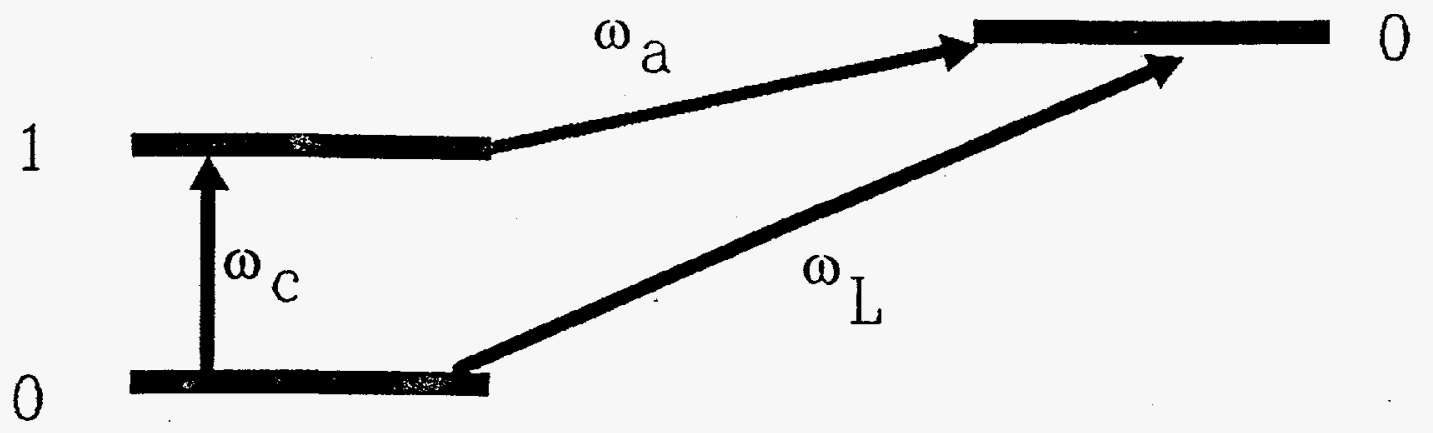




\section{Antiproton Catching Trap}

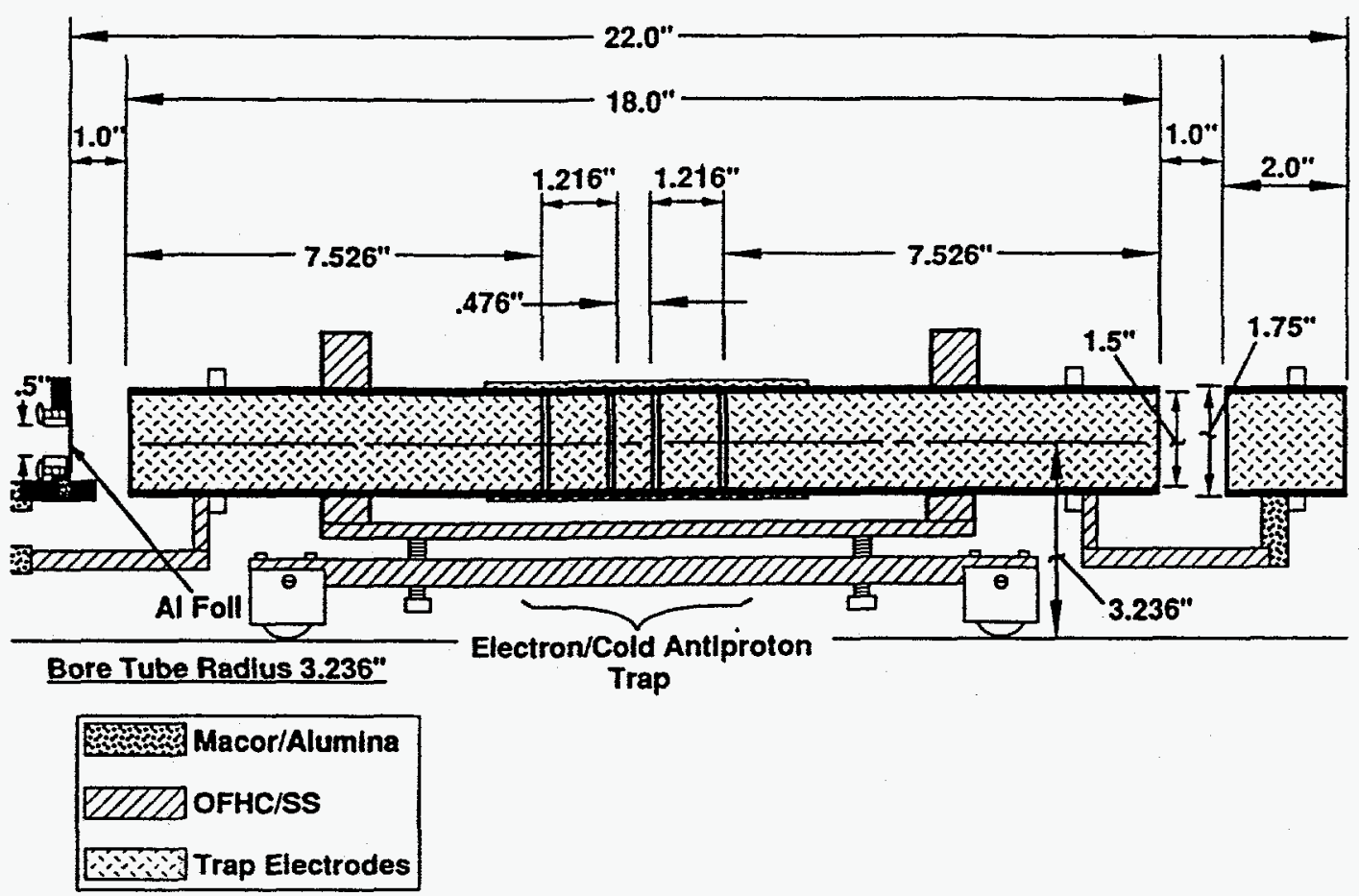

Energy Spectrum of Antiprotons released from Trap

(Channel $0=28.5 \mathrm{keV}$, Channel $600=0 \mathrm{eV}$ )

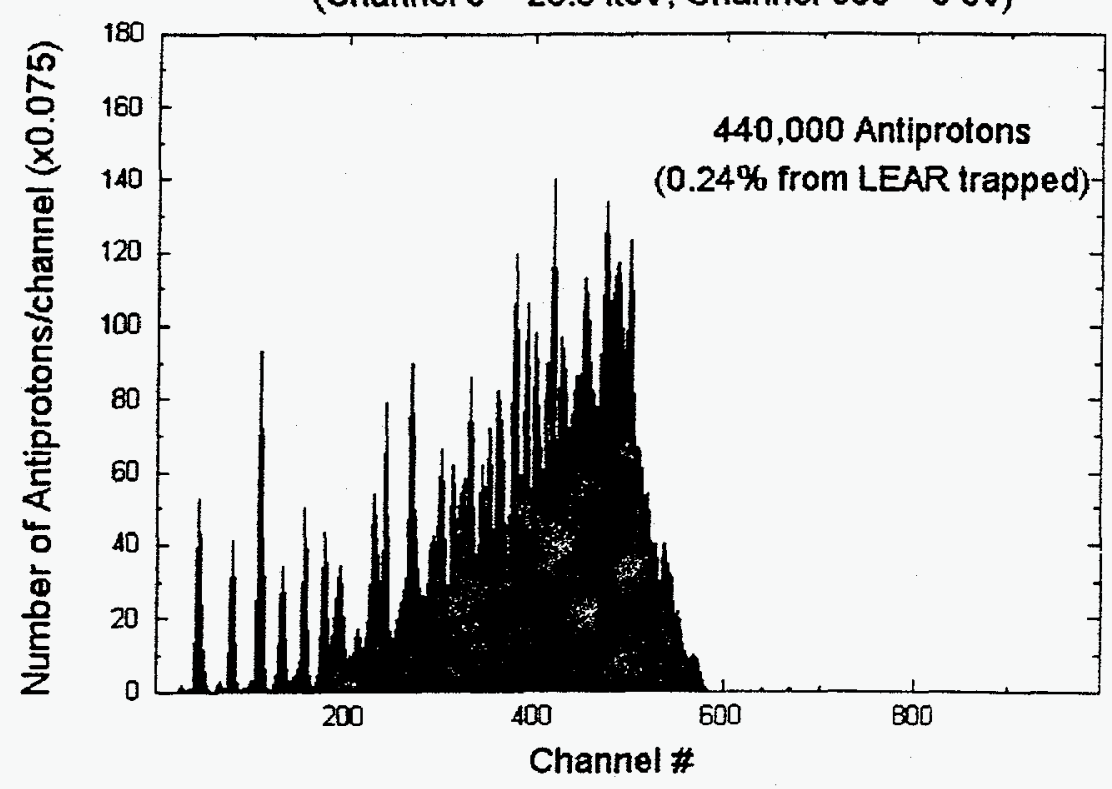




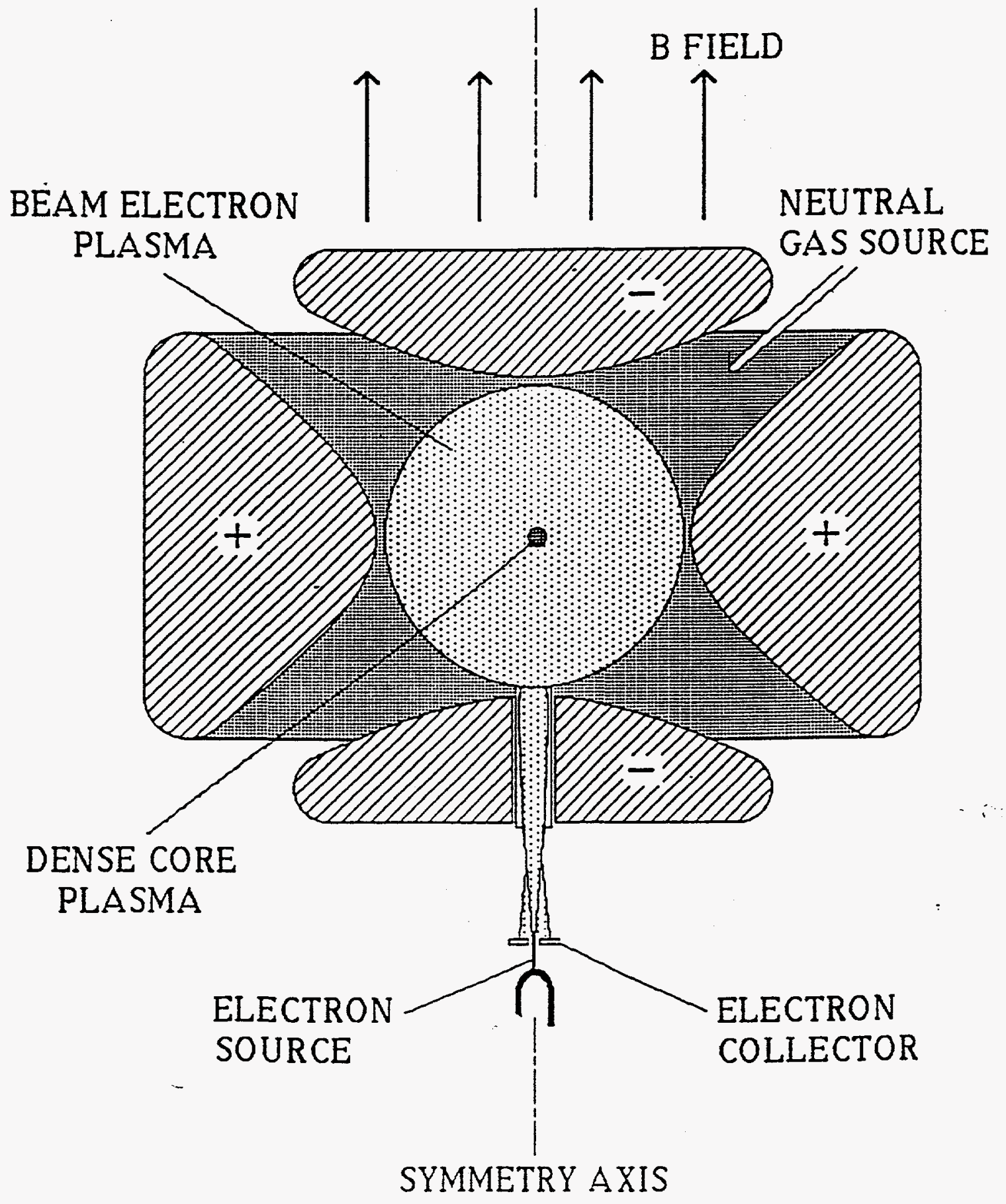

